\title{
Consumption of ultra-processed foods and cancer risk: results from NutriNet-Santé prospective cohort
}

\author{
Thibault Fiolet, ${ }^{1}$ Bernard Srour, ${ }^{1}$ Laury Sellem, ${ }^{1}$ Emmanuelle Kesse-Guyot, ${ }^{1}$ Benjamin Allès, ${ }^{1}$ \\ Caroline Méjean, ${ }^{2}$ Mélanie Deschasaux, ${ }^{1}$ Philippine Fassier, ${ }^{1}$ Paule Latino-Martel, ${ }^{1}$ \\ Marie Beslay, ${ }^{1}$ Serge Hercberg, ${ }^{1,4}$ Céline Lavalette, ${ }^{1}$ Carlos A Monteiro, ${ }^{3}$ Chantal Julia, ${ }^{1,4}$ \\ Mathilde Touvier ${ }^{1}$
}

${ }^{1}$ Sorbonne Paris Cité

Epidemiology and Statistics

Research Center (CRESS),

Inserm U1153, Inra U1125,

Cnam, Paris 13 University,

Nutritional Epidemiology

Research Team (EREN),

Bobigny, France

${ }^{2}$ INRA, UMR 1110 MOISA,

34000 Montpellier, France

${ }^{3}$ Department of Nutrition,

School of Public Health,

University of São Paulo, São

Paulo 01246-904, Brazil

${ }^{4}$ Public Health Department,

Avicenne Hospital, AP-HP,

Bobigny, France

Correspondence to: B Srour

b.srour@eren.smbh.

univ-paris13.fr

Additional material is published online only. To view please visit the journal online.

Cite this as: BMJ 2018;360:k322 http://dx.doi.org/10.1136/bmj.k322

Accepted: 10 January 2018

\section{ABSTRACT}

OBJECTIVE

To assess the prospective associations between consumption of ultra-processed food and risk of cancer.

\section{DESIGN}

Population based cohort study.

\section{SETTING AND PARTICIPANTS}

104980 participants aged at least 18 years (median age 42.8 years) from the French NutriNet-Santé cohort (2009-17). Dietary intakes were collected using repeated 24 hour dietary records, designed to register participants' usual consumption for 3300 different food items. These were categorised according to their degree of processing by the NOVA classification.

\section{MAIN OUTCOME MEASURES}

Associations between ultra-processed food intake and risk of overall, breast, prostate, and colorectal cancer assessed by multivariable Cox proportional hazard models adjusted for known risk factors.

\section{RESULTS}

Ultra-processed food intake was associated with higher overall cancer risk ( $n=2228$ cases; hazard ratio for a $10 \%$ increment in the proportion of ultraprocessed food in the diet 1.12 (95\% confidence interval 1.06 to 1.18); P for trend 0.001 ) and breast cancer risk ( $\mathrm{n}=739$ cases; hazard ratio 1.11 (1.02 to 1.22); $P$ for trend=0.02). These results remained

\section{WHAT IS ALREADY KNOWN ON THIS TOPIC}

Ultra-processed foods are often characterised by lower nutritional quality and the presence of additives, substances from packaging in contact with food, and compounds formed during production, processing, and storage

A few studies have observed ultra-processed food intake to be associated with a higher incidence of dyslipidaemia in Brazilian children and higher risks of overweight, obesity, and hypertension in Spanish university students

Although epidemiological data relating to cancer risk are lacking, mechanistic studies suggest potential carcinogenic effects of several components commonly found in ultra-processed foods

\section{WHAT THIS STUDY ADDS}

This study assessed the associations between ultra-processed food consumption and risk of cancer in a large prospective cohort

A $10 \%$ increase in the proportion of ultra-processed foods in the diet was associated with a significant increase of more than $10 \%$ in the risks of overall and breast cancer

If confirmed in other populations and settings, these results suggest that the rapidly increasing consumption of ultra-processed foods may drive an increasing burden of cancer in the next decades

statistically significant after adjustment for several markers of the nutritional quality of the diet (lipid, sodium, and carbohydrate intakes and/or a Western pattern derived by principal component analysis).

\section{CONCLUSIONS}

In this large prospective study, a $10 \%$ increase in the proportion of ultra-processed foods in the diet was associated with a significant increase of greater than $10 \%$ in risks of overall and breast cancer. Further studies are needed to better understand the relative effect of the various dimensions of processing (nutritional composition, food additives, contact materials, and neoformed contaminants) in these associations.

\section{STUDY REGISTRATION}

Clinicaltrials.gov NCT03335644.

\section{Introduction}

Cancer represents a major worldwide burden, with 14.1 million new cases diagnosed in 2012. ${ }^{1}$ According to the World Cancer Research Fund/American Institute for Cancer Research, about a third of the most common neoplasms could be avoided by changing lifestyle and dietary habits in developed countries. ${ }^{2}$ Therefore, reaching a balanced and diversified diet (along with avoidance of tobacco use and reduction in alcohol intake) should be considered one of the most important modifiable risk factors in the primary prevention of cancer. $^{3}$

At the same time, during the past decades, diets in many countries have shifted towards a dramatic increase in consumption of ultra-processed foods. ${ }^{4-8}$ After undergoing multiple physical, biological, and/ or chemical processes, these food products are conceived to be microbiologically safe, convenient, highly palatable, and affordable. ${ }^{9} 10$ Several surveys (in Europe, the US, Canada, New Zealand, and Brazil) assessing individual food intake, household food expenses, or supermarket sales have suggested that ultra-processed food products contribute to between $25 \%$ and $50 \%$ of total daily energy intake. ${ }^{10-18}$

This dietary trend may be concerning and deserves investigation. Several characteristics of ultraprocessed foods may be involved in causing disease, particularly cancer. Firstly, ultra-processed foods often have a higher content of total fat, saturated fat, and added sugar and salt, along with a lower fibre and vitamin density. ${ }^{10-17} 19$ Beyond nutritional composition, neoformed contaminants, some of which have carcinogenic properties (such as acrylamide, heterocyclic amines, and polycyclic aromatic 
hydrocarbons), are present in heat treated processed food products as a result of the Maillard reaction. ${ }^{20}$ Secondly, the packaging of ultra-processed foods may contain some materials in contact with food for which carcinogenic and endocrine disruptor properties have been postulated, such as bisphenol A. ${ }^{21}$ Finally, ultra-processed foods contain authorised, ${ }^{22}$ but controversial, food additives such as sodium nitrite in processed meat or titanium dioxide $\left(\mathrm{TiO}_{2}\right.$, white food pigment), for which carcinogenicity has been suggested in animal or cellular models. ${ }^{2324}$

Studying potential effects on health of ultraprocessed foods is a very recent field of research, facilitated by the development of the NOVA classification of products according to their degree of food processing. ${ }^{9}$ Nevertheless, epidemiological evidence linking intake of ultra-processed food to risk of disease is still very scarce and mostly based on cross sectional and ecological studies. ${ }^{25-27}$ The few studies performed observed that ultra-processed food intake was associated with a higher incidence of dyslipidaemia in Brazilian children and higher risks of overweight, obesity, and hypertension in a prospective cohort of Spanish university students. ${ }^{28-30}$

To our knowledge, this prospective study was the first to evaluate the association between the consumption of ultra-processed food products and the incidence of cancer, based on a large cohort study with detailed and up to date assessment of dietary intake.

\section{Methods \\ Study population}

The NutriNet-Santé study is an ongoing web based cohort launched in 2009 in France with the objective of studying the associations between nutrition and health, as well as the determinants of dietary behaviours and nutritional status. This cohort has been previously described in detail. ${ }^{31}$ Briefly, participants aged over 18 years with access to the internet have been continuously recruited from among the general population since May 2009 by means of vast multimedia campaigns. All questionnaires are completed online using a dedicated website (www.etude-nutrinet-sante.fr). Participants are followed using an online platform connected to their email address. They can change their email address, phone number, or postal address at any time on the NutriNet-Santé website. Newsletters and alerts about new questionnaires are sent by email. In case of an "undelivered email” problem, participants are contacted by telephone and then by regular mail. The NutriNet-Santé study is conducted according to the Declaration of Helsinki guidelines, and electronic informed consent is obtained from each participant.

\section{Data collection}

At inclusion, participants completed a set of five questionnaires related to sociodemographic and lifestyle characteristics (for example, date of birth, sex, occupation, educational level, smoking status, number of children), ${ }^{32}$ anthropometry (height, weight), dietary intakes (see below), ${ }^{33} 34$ physical activity (validated seven day International Physical Activity Questionnaire (IPAQ)), ${ }^{35}$ and health status (personal and family history of diseases, drug use including use of hormonal treatment for menopause and oral contraceptives, and menopausal status).

Participants were invited to complete a series of three non-consecutive, validated, web based 24 hour dietary records every six months (to vary the season of completion), randomly assigned over a two week period (two weekdays and one weekend day). ${ }^{36-38}$ To be included in the nutrition component of the NutriNetSanté cohort, only two dietary records were mandatory. We did not exclude participants if they did not complete all optional questionnaires. We averaged mean dietary intakes from all the 24 hour dietary records available during the first two years of each participant's followup and considered these as baseline usual dietary intakes in this prospective analysis. The NutriNet-Santé web based, self administered 24 hour dietary records have been tested and validated against an interview by a trained dietitian and against blood and urinary biomarkers. ${ }^{36} 37$ Participants used the dedicated web interface to declare all food and drinks consumed during a 24 hour period for each of the three main meals (breakfast, lunch, dinner) and any other eating occasion. Portion sizes were estimated using previously validated photographs or usual containers. ${ }^{39}$ We identified dietary under-reporting on the basis of the method proposed by Black, using the basal metabolic rate and Goldberg cutoff, and excluded under-reporters of energy intake. ${ }^{40}$ We calculated mean daily alcohol, micronutrient and macronutrient, and energy intake by using the NutriNetSanté food composition database, which contains more than 3300 different items. ${ }^{41}$ We estimated amounts consumed from composite dishes by using French recipes validated by nutrition professionals. Sodium intake was assessed via a specific module included in the 24 hour records, taking into account native sodium in foods, salt added during the cooking, and salt added on the plate. It has been validated against sodium urinary excretion biomarkers. ${ }^{37}$

\section{Degree of food processing}

We categorised all food and drink items of the NutriNet-Santé composition table into one of the four food groups in NOVA, a food classification system based on the extent and purpose of industrial food processing. 9243 This study primarily focused on the "ultra-processed foods" NOVA group. This group includes mass produced packaged breads and buns; sweet or savoury packaged snacks; industrialised confectionery and desserts; sodas and sweetened drinks; meat balls, poultry and fish nuggets, and other reconstituted meat products transformed with addition of preservatives other than salt (for example, nitrites); instant noodles and soups; frozen or shelf stable ready meals; and other food products made mostly or entirely from sugar, oils and fats, and other substances not commonly used in culinary preparations such as hydrogenated oils, modified starches, and protein isolates. Industrial processes 
notably include hydrogenation, hydrolysis, extruding, moulding, reshaping, and pre-processing by frying. Flavouring agents, colours, emulsifiers, humectants, non-sugar sweeteners, and other cosmetic additives are often added to these products to imitate sensorial properties of unprocessed or minimally processed foods and their culinary preparations or to disguise undesirable qualities of the final product.

The ultra-processed food group is defined by opposition to the other NOVA groups: "unprocessed or minimally processed foods" (fresh, dried, ground, chilled, frozen, pasteurised, or fermented staple foods such as fruits, vegetables, pulses, rice, pasta, eggs, meat, fish, or milk), "processed culinary ingredients" (salt, vegetable oils, butter, sugar, and other substances extracted from foods and used in kitchens to transform unprocessed or minimally processed foods into culinary preparations), and "processed foods" (canned vegetables with added salt, sugar coated dried fruits, meat products preserved only by salting, cheeses, freshly made unpackaged breads, and other products manufactured with the addition of salt, sugar, or other substances of the "processed culinary ingredients" group). As previously described, ${ }^{44}$ we identified homemade and artisanal food preparations, decomposed them using standardised recipes, and applied the NOVA classification to their ingredients. Precision and examples are shown in appendix 1.

\section{Case ascertainment}

Participants self declared health events through the yearly health status questionnaire, through a specific check-up questionnaire for health events (every three months), or at any time through a specific interface on the study website. For each incident cancer declared, a physician from the study team contacted participants and asked them to provide any relevant medical records. If necessary, the study physicians contacted the patient's physician and/or hospitals to collect additional information. Afterwards, an expert committee of physicians reviewed all medical data. Our research team was the first in France to obtain the authorisation by decree in the Council of State (No 2013-175) to link data from our cohorts to medicoadministrative databases of the national health insurance system (SNIIRAM databases). We therefore completed declared health events with the information from these databases, thereby limiting any potential bias due to participants with cancer who may not report their disease to the study investigators. Lastly, we used an additional linkage to the French national cause specific mortality registry (CépiDC) to detect deaths and potentially missed cases of cancer for deceased participants. We classified cancer cases by using the international classification of diseases, 10th revision (ICD-10). In this study, we considered all first primary cancers diagnosed between the inclusion date and 1 January 2017 to be cases, except for basal cell skin carcinoma, which we did not consider as cancer.

We obtained medical records for more than $90 \%$ of cancer cases. Because of the high validity of self reports $(95 \%$ of self reported cancers for which a medical record was obtained were confirmed by our physicians), we included as cases all participants who self reported incident cancers, unless they were identified as non-case participants by a pathology report, in which case we classified them as non-cases.

\section{Statistical analysis}

Up to 1 January 2017, we included 104980 participants without cancer at baseline who provided at least two valid 24 hour dietary records during their two first years of follow-up. The flowchart is in appendix 2. For each participant, we calculated the proportion (percentage g/day) of ultra-processed foods in the total diet. We determined the proportion of ultra-processed foods in the diet by calculating a weight ratio rather than an energy ratio to take into account processed foods that do not provide any energy (in particular artificially sweetened drinks) and non-nutritional factors related to food processing (for example, neoformed contaminants, food additives, and alterations to the structure of raw foods). For all covariates except physical activity, less than 5\% of values were missing and were imputed to the modal value (for categorical variables) or to the median (for continuous variables). Corresponding values are provided in the footnote to table 1 . The proportion of missing values was higher for physical activity (14\%), as the answers to all IPAQ questions were needed to calculate the score. To avoid massive imputation for a non-negligible number of participants or exclusion of those with missing data and risk of selection bias, we included a missing class into the models for this variable. We examined differences in participants' baseline characteristics between sex specific quarters of the proportion of ultra-processed food in the diet by using analysis of variance or $\chi^{2}$ tests wherever appropriate. We used Cox proportional hazards models with age as the primary timescale to evaluate the association between the proportion of ultra-processed foods in the diet (coded as a continuous variable or as sex specific quarters) and incidence of overall, breast, prostate, and colorectal cancer. In these models, cancers at other locations than the one studied were censored at the date of diagnosis (that is, we considered them to be non-cases for the cancer of interest and they contributed person years until the date of diagnosis of their cancer). We estimated hazard ratios and 95\% confidence intervals with the lowest quarter as the reference category. We generated log-log (survival) versus log-time plots to confirm risk proportionality assumptions. We tested for linear trend by using the ordinal score on sex specific quarters of ultra-processed food. Participants contributed person time until the date of diagnosis of cancer, the date of last completed questionnaire, the date of death, or 1 January 2017, whichever occurred first. Breast cancer analyses were additionally stratified by menopausal status. For these, women contributed person time to the "premenopause model" until their age at menopause and to the "postmenopause model" from their age at menopause. We determined 
Table 1 | Baseline characteristics of study population according to sex specific quarters of ultra-processed food consumption ( $n=104980$ ), NutriNetSanté cohort, France, 2009-17*. Values are numbers (percentages) unless stated otherwise

\begin{tabular}{|c|c|c|c|c|c|c|}
\hline \multirow[b]{2}{*}{ Characteristics } & \multirow[b]{2}{*}{ All participants } & \multicolumn{4}{|c|}{ Quarters of ultra-processed food consumptiont } & \multirow[b]{2}{*}{$P$ for trend $\neq$} \\
\hline & & $1(n=26244)$ & $2(n=26245)$ & $3(n=26246)$ & $4(n=26245)$ & \\
\hline Mean (SD) age, years & $42.8(14.8)$ & $47.9(13.5)$ & $45.0(14.0)$ & $42.0(14.4)$ & $36.5(13.6)$ & $<0.001$ \\
\hline Female sex & $82159(78.3)$ & $20539(78.3)$ & $20540(78.3)$ & $20541(78.3)$ & $20542(78.3)$ & - \\
\hline Mean (SD) height, $\mathrm{cm}$ & $166.8(8.1)$ & $166.3(8.0)$ & $166.7(8.0)$ & $167.0(8.1)$ & $167.3(8.2)$ & $<0.001$ \\
\hline Mean (SD) body mass index & $23.8(4.6)$ & $23.8(4.3)$ & $23.8(4.4)$ & $23.8(4.5)$ & $23.8(5.0)$ & 0.9 \\
\hline Family history of cancer§ & $35668(34.0)$ & $10542(40.2)$ & $9624(36.7)$ & $8625(32.9)$ & $6877(26.2)$ & $<0.001$ \\
\hline \multicolumn{6}{|l|}{ Higher education: } & \multirow{4}{*}{0.01} \\
\hline No & $19357(18.4)$ & $5154(19.6)$ & $4961(18.9)$ & $4637(17.7)$ & $4605(17.6)$ & \\
\hline Yes, $<2$ years & $18076(17.2)$ & $3938(15.0)$ & $4091(15.6)$ & $4426(16.9)$ & $5621(21.4)$ & \\
\hline Yes, $\geq 2$ years & $67547(64.3)$ & $17152(65.4)$ & $17193(65.5)$ & $17183(65.5)$ & $16019(61.0)$ & \\
\hline \multicolumn{6}{|l|}{ Smoking status: } & \multirow{3}{*}{$<0.001$} \\
\hline Current & $17763(16.9)$ & $4127(15.7)$ & $4065(15.5)$ & $4266(16.3)$ & $5305(20.2)$ & \\
\hline Never/former & $87217(83.1)$ & $22117(84.3)$ & $22180(84.5)$ & $21980(83.8)$ & $20940(79.8)$ & \\
\hline \multicolumn{6}{|l|}{ IPAQ physical activity level:@ } & \multirow{4}{*}{$<0.001$} \\
\hline High & $29603(28.2)$ & $8753(33.4)$ & $7762(29.6)$ & $6983(26.6)$ & $6105(23.3)$ & \\
\hline Moderate & $38874(37.0)$ & $9620(36.7)$ & $9953(37.9)$ & $9814(37.4)$ & $9487(36.2)$ & \\
\hline Low & $21888(20.9)$ & $4407(16.8)$ & $5152(19.6)$ & $5839(22.3)$ & $6490(24.7)$ & \\
\hline Mean (SD) energy intake without alcohol, kcal/d & $1879.0(473.7)$ & $1810.6(454.1)$ & $1881.1(457.7)$ & $1908.5(472.3)$ & $1915.8(501.8)$ & $<0.001$ \\
\hline Mean (SD) alcohol intake, $\mathrm{g} / \mathrm{d}$ & $7.8(11.9)$ & $9.3(13.3)$ & $8.5(11.9)$ & $7.5(11.3)$ & $5.9(10.5)$ & $<0.001$ \\
\hline Mean (SD) total lipid intake, $\mathrm{g} / \mathrm{d}$ & $80.5(25.5)$ & $76.0(24.3)$ & $80.3(24.4)$ & $82.1(25.3)$ & $83.4(27.3)$ & $<0.001$ \\
\hline Mean (SD) carbohydrate intake, $\mathrm{g} / \mathrm{d}$ & $195.4(57.9)$ & $184.6(57.8)$ & $193.9(55.3)$ & $199.3(56.6)$ & $203.6(60.2)$ & $<0.001$ \\
\hline Mean (SD) sodium intake, $\mathrm{mg} / \mathrm{d}$ & $2700.1(893.1)$ & $2589.3(881.6)$ & $2731.8(871.0)$ & $2761.9(884.1)$ & $2717.7(925.0)$ & $<0.001$ \\
\hline Mean (SD) No of children & $1.3(1.2)$ & $1.6(1.2)$ & $1.4(1.2)$ & $1.3(1.2)$ & $1.0(1.2)$ & $<0.001$ \\
\hline \multicolumn{6}{|l|}{ Menopausal status: ${ }^{* \star}$} & \multirow{4}{*}{$<0.001$} \\
\hline Premenopausal & $57408(69.9)$ & $11797(57.4)$ & $13497(65.7)$ & $14961(72.8)$ & $17153(83.5)$ & \\
\hline Perimenopausal & $4282(5.2)$ & $1471(7.2)$ & $1148(5.6)$ & $997(4.9)$ & $666(3.2)$ & \\
\hline Postmenopausal & $20469(24.9)$ & $7271(35.4)$ & $5895(28.7)$ & $4582(22.3)$ & $2721(13.3)$ & \\
\hline Use of hormonal treatment for menopause ${ }^{\star \star}$ & $4324(5.3)$ & $1602(7.8)$ & $1242(6.1)$ & $932(4.5)$ & $548(2.7)$ & $<0.001$ \\
\hline Oral contraception ${ }^{\star \star}$ & $23073(22.0)$ & $3779(14.4)$ & $4990(19.0)$ & $6209(23.7)$ & $8095(30.8)$ & $<0.001$ \\
\hline Mean (SD) ultra-processed food, \% & $18.7(10.1)$ & $8.5(2.5)$ & $14.3(1.4)$ & $19.8(1.9)$ & $32.3(9.8)$ & - \\
\hline
\end{tabular}

IPAQ=International Physical Activity Questionnaire.

*For all covariates except physical activity, a very low proportion of values were missing (0-5\%); these were replaced by modal value in study population: " $\geq 2$ years of higher education" for educational level, 0 for No of biological children, 22.9 for body mass index, $166 \mathrm{~cm}$ for height, and non-smoker for smoking status.

tSex specific quarters of proportion of ultra-processed food intake in total quantity of food consumed; sex specific cut-offs for quarters of ultra-processed proportions were $11.8 \%, 16.8 \%$, and $23.3 \%$ in men and $11.8 \%, 16.8 \%$, and $23.4 \%$ in women.

‡P value for comparison between sex specific quarters of ultra-processed food consumption, by Fisher test or $\mathrm{x}^{2}$ test where appropriate.

$\S$ Among first degree relatives.

|Available for 90365 participants; participants were categorised into "high," "moderate," and "low" categories according to IPAQ guidelines.

**Among women.

age at menopause by using the yearly health status questionnaires completed during follow-up.

Models were adjusted for age (timescale), sex, body mass index $\left(\mathrm{kg} / \mathrm{m}^{2}\right.$, continuous), height ( $\mathrm{cm}$, continuous), physical activity (high, moderate, low, calculated according to IPAQ recommendations ${ }^{35}$ ), smoking status (never or former smokers, current smokers), number of 24 hour dietary records (continuous), alcohol intake (g/d, continuous), energy intake (without alcohol, kcal/d, continuous), family history of cancer (yes/no), and educational level (less than high school degree, less than two years after high school degree, two or more years after high school degree). For breast cancer analyses, we made additional adjustments for the number of biological children (continuous), menopausal status at baseline (menopausal/perimenopausal/nonmenopausal), hormonal treatment for menopause at baseline (for postmenopausal analyses, yes/no), and oral contraception use at baseline (for premenopausal analyses, yes/no) (model 1=main model). To test for the potential influence of the nutritional quality of the diet in the relation between intake of ultra-processed food and risk of cancer, this model was additionally adjusted for lipid, sodium, and carbohydrate intakes (model 2), for a Western dietary pattern derived from principal component analysis (model 3) (details in appendix 3), or for all these nutritional factors together (model 4). In addition, we did mediation analyses according to the method proposed by Lange et al to evaluate the direct and indirect effect of the relation between the exposure and the outcome through the following nutritional mediators: intakes of sodium, total lipids, saturated, mono-unsaturated and polyunsaturated fatty acids, carbohydrates, and a Westerntype dietary pattern. ${ }^{45}$ The methods are described in appendix 4.

We did sensitivity analyses based on model 1 by excluding cases of cancer diagnosed during the first two years of each participant's follow-up to avoid reverse causality bias, testing sex specific fifths of the proportion of ultra-processed foods in the diet instead of sex specific quarters, and testing further adjustments for prevalent depression at baseline (yes/no), dietary supplement use at baseline (yes/no), healthy dietary pattern (continuous, details in appendix 3), number of cigarettes smoked in pack years (continuous), overall fruit and vegetable consumption (continuous), and 
season of inclusion in the cohort (spring/summer/ autumn/winter). We also investigated the association between ultra-processed food and overall cancer risk separately in different strata of the population: men, women, younger adults (under 40 years), older adults (40 years or over), smokers, non-smokers, participants with a high level of physical activity, and those with a low to moderate level of physical activity. We also tested models after restriction of the study population to the participants with at least six 24 hour dietary records during the first two years of follow-up. Similarly, we tested models including all participants with at least one 24 hour dietary record during the first two years of follow-up. We also tested associations between the quantity $(\mathrm{g} / \mathrm{d}$ ) of each ultra-processed food group and risk of cancer.

Secondary analyses tested the associations between the proportion in the diet of each of the three other NOVA categories of food processing (continuous) and risk of cancer, using multivariate Cox models adjusted for model 1 covariates. All tests were two sided, with $\mathrm{P}<0.05$ considered to be statistically significant. We used SAS version 9.4 for the analyses.

\section{Patient involvement}

The research question developed in this article corresponds to a strong concern of the participants involved in the NutriNet-Sante cohort and of the public in general. The results of this study will be disseminated to the NutriNet-Sante participants through the cohort website, public seminars, and a press release.

\section{Results}

A total of 104980 participants (22 821 (21.7\%) men and $82159(78.3 \%)$ women) were included in the study. The mean age of participants was 42.8 (SD 14.8, range 18.0-72.8) years. The mean number of dietary records per participant over their first two years of follow-up was 5.4 (SD 2.9); the minimum was 2, but it represented only $7.2 \%(7558 / 104980)$ of the participants. After the launching of the study by the end of May 2009, half of the records were filled between June and November and the other half between December and May. Table 1 shows the main baseline characteristics of participants according to quarters of the proportion of ultra-processed foods in the diet. Compared with the lowest quarter, participants in the highest quarter of ultra-processed food intake tended to be younger, current smokers, and less educated, with less family history of cancer and a lower physical activity level. Furthermore, they had higher intakes of energy, lipids, carbohydrates, and sodium, along with lower alcohol intake. Although there was a higher proportion of women than men in this cohort, the contribution of ultra-processed foods to the overall diet was very similar between men and women (18.74\% for men and $18.71 \%$ for women; $\mathrm{P}=0.7)$. The distribution of the proportion of ultra-processed food in the diet in the study population is shown in appendix 5. Main food groups contributing to ultra-processed food intake were sugary products (26\%) and drinks (20\%),

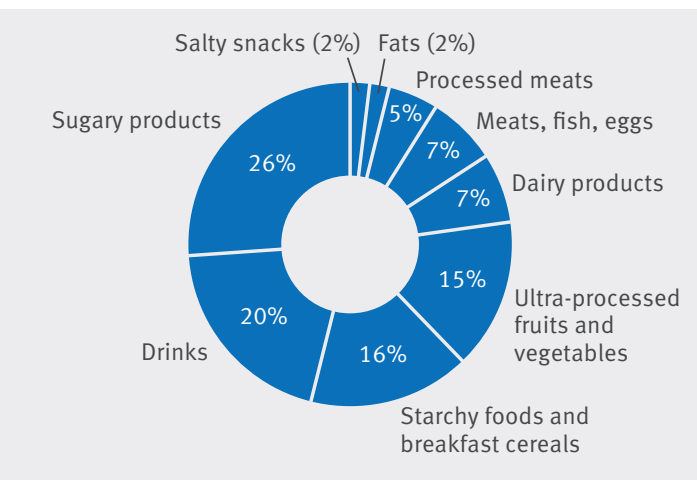

Fig 1 | Relative contribution of each food group to ultra-processed food consumption in diet

followed by starchy foods and breakfast cereals (16\%) and ultra-processed fruits and vegetables (15\%) (fig 1).

During follow-up (426362 person years, median follow-up time five years), 2228 first incident cases of cancer were diagnosed and validated, among which were 739 breast cancers (264 premenopausal, 475 postmenopausal), 281 prostate cancers, and 153 colorectal cancers. Among these 2228 cases, 108 (4.8\%) were identified during mortality followup with the national CépiDC database. The dropout rate in the NutriNet-Santé cohort was $6.7 \%$. Table 2 shows associations between the proportion of ultraprocessed foods in the diet and risks of overall, breast, prostate, and colorectal cancer. Figure 2 shows the corresponding cumulative incidence curves. In model 1, ultra-processed food intake was associated with increased risks of overall cancer (hazard ratio for a 10 point increment in the proportion of ultra-processed foods in the diet 1.12 (95\% confidence interval 1.06 to 1.18), $\mathrm{P}<0.001)$ and breast cancer (1.11 (1.02 to 1.22), $\mathrm{P}=0.02)$. The latter association was more specifically observed for postmenopausal breast cancer $(\mathrm{P}=0.04)$ but not for premenopausal breast cancer $(\mathrm{P}=0.2)$. The association with overall cancer risk was statistically significant in all strata of the population investigated, after adjustment for model 1 covariates: in men (hazard ratio for a 10 point increment in the proportion of ultra-processed foods in the diet 1.12 (1.02 to 1.24), $\mathrm{P}=0.02,663$ cases and 22158 non-cases), in women (1.13 (1.06 to 1.20), $\mathrm{P}<0.001,1565$ cases and 80594 non-cases), in younger adults ( $<40$ years old 1.21 (1.09 to 1.35), $\mathrm{P}<0.001,287$ cases and 48627 noncases), in older adults ( $\geq 40$ years old, 1.09 (1.03 to 1.16), $\mathrm{P}=0.03$, 1941 cases and 54485 non-cases), in smokers (including adjustment for pack years of cigarettes smoked 1.18 (1.04 to 1.33), $\mathrm{P}=0.01,255$ cases and 15355 non-cases), in non-smokers (1.11 (1.05 to 1.17), $\mathrm{P}<0.001,1943$ cases and 85219 noncases), in participants with low to moderate levels of physical activity (1.07 (1.00 to 1.15), P=0.04, 1216 cases and 59546 non-cases), and in those with a high level of physical activity (1.19 (1.09 to 1.30), $\mathrm{P}<0.001$, 744 cases and 28859 non-cases).

More specifically, ultra-processed fats and sauces $(\mathrm{P}=0.002)$ and sugary products $(\mathrm{P}=0.03)$ and drinks 


\begin{tabular}{|c|c|c|c|c|c|c|c|}
\hline & \multicolumn{7}{|c|}{ Proportion of ultra-processed food intake in the diet } \\
\hline & \multirow[b]{2}{*}{ Continuoust } & & \multicolumn{5}{|c|}{ Sex specific quartersł } \\
\hline & & & 1 & 2 & 3 & 4 & \\
\hline & $\mathrm{HR}(95 \% \mathrm{Cl})$ & $P$ for trend & $\mathrm{HR}$ & $\mathrm{HR}(95 \% \mathrm{Cl})$ & $\mathrm{HR}(95 \% \mathrm{Cl})$ & P for trend $\mathrm{HR}(95 \% \mathrm{Cl})$ & \\
\hline \multicolumn{8}{|l|}{ All cancers } \\
\hline No of cases/non-cases & $2228 / 102752$ & & $712 / 25532$ & $607 / 25638$ & $541 / 25705$ & $368 / 25877$ & \\
\hline Model 1 & $1.12(1.06$ to 1.18$)$ & $<0.001$ & 1 & $0.99(0.89$ to 1.11$)$ & $1.10(0.99$ to 1.24$)$ & $1.21(1.06$ to 1.38$)$ & 0.002 \\
\hline Model 2 & $1.12(1.07$ to 1.18$)$ & $<0.001$ & 1 & $1.00(0.90$ to 1.11$)$ & $1.11(0.99$ to 1.25$)$ & $1.23(1.08$ to 1.40$)$ & 0.001 \\
\hline Model 3 & $1.12(1.06$ to 1.18$)$ & $<0.001$ & 1 & 0.99 (0.89 to 1.11$)$ & $1.01(0.98$ to 1.23$)$ & $1.21(1.06$ to 1.38$)$ & 0.002 \\
\hline Model 4 & $1.13(1.07$ to 1.18$)$ & $<0.001$ & 1 & $1.00(0.90$ to 1.11$)$ & $1.11(0.99$ to 1.24$)$ & $1.23(1.08$ to 1.40$)$ & 0.001 \\
\hline \multicolumn{8}{|l|}{ Prostate cancer } \\
\hline No of cases/non-cases & $281 / 22540$ & & $96 / 5609$ & $96 / 5609$ & $59 / 5647$ & $30 / 5675$ & \\
\hline Model 1 & $0.98(0.83$ to 1.16$)$ & 0.8 & 1 & 1.18 (0.89 to 1.57$)$ & 0.95 (0.69 to 1.32$)$ & $0.93(0.61$ to 1.40$)$ & 0.6 \\
\hline Model 2 & $0.98(0.83$ to 1.16$)$ & 0.8 & 1 & 1.18 (0.89 to 1.57$)$ & 0.95 (0.69 to 1.32$)$ & $0.93(0.61$ to 1.40$)$ & 0.6 \\
\hline Model 3 & $0.98(0.83$ to 1.15$)$ & 0.8 & 1 & 1.18 (0.89 to 1.56$)$ & 0.95 (0.68 to 1.31$)$ & 0.92 (0.61 to 1.39$)$ & 0.6 \\
\hline Model 4 & $0.98(0.83$ to 1.16$)$ & 0.8 & 1 & 1.18 (0.89 to 1.57$)$ & 0.95 (0.68 to 1.32) & $0.93(0.61$ to 1.40$)$ & 0.6 \\
\hline \multicolumn{8}{|l|}{ Colorectal cancer } \\
\hline No of cases/non-cases & $153 / 104827$ & & $48 / 26196$ & $43 / 26202$ & $36 / 26210$ & $26 / 26219$ & \\
\hline Model 1 & $1.13(0.92$ to 1.38$)$ & 0.2 & 1 & $1.10(0.72$ to 1.66$)$ & $1.17(0.76$ to 1.81$)$ & $1.49(0.92$ to 2.43$)$ & 0.1 \\
\hline Model 2 & $1.16(0.95$ to 1.42$)$ & 0.1 & 1 & $1.12(0.74$ to 1.70$)$ & $1.22(0.79$ to 1.90$)$ & $1.59(0.97$ to 2.60$)$ & 0.07 \\
\hline Model 3 & $1.13(0.92$ to 1.38$)$ & 0.2 & 1 & $1.09(0.92$ to 1.38$)$ & $1.16(0.75$ to 1.80$)$ & $1.48(0.91$ to 2.41$)$ & 0.1 \\
\hline Model 4 & $1.16(0.95$ to 1.42$)$ & 0.1 & 1 & $1.12(0.74$ to 1.70$)$ & $1.22(0.79$ to 1.89$)$ & 1.23 (1.08 to 1.40$)$ & 0.07 \\
\hline \multicolumn{8}{|l|}{ Breast cancer } \\
\hline No of cases/non-cases & $739 / 81420$ & & $247 / 20292$ & $202 / 20338$ & $179 / 20361$ & $111 / 20429$ & \\
\hline Model 1 & 1.11 (1.02 to 1.22$)$ & 0.02 & 1 & 0.97 (0.81 to 1.17$)$ & 1.10 (0.90 to 1.34$)$ & 1.14 (0.91 to 1.44$)$ & 0.2 \\
\hline Model 2 & 1.11 (1.01 to 1.21$)$ & 0.03 & 1 & $0.96(0.80$ to 1.16$)$ & 1.09 (0.89 to 1.32$)$ & $1.12(0.89$ to 1.42$)$ & 0.2 \\
\hline Model 3 & 1.11 (1.02 to 1.22$)$ & 0.02 & 1 & $0.97(0.80$ to 1.17$)$ & $1.09(0.90$ to 1.33$)$ & $1.14(0.91$ to 1.44$)$ & 0.2 \\
\hline Model 4 & 1.11 (1.01 to 1.21$)$ & 0.03 & 1 & $0.96(0.80$ to 1.16$)$ & $1.08(0.89$ to 1.32$)$ & $1.13(0.89$ to 1.42$)$ & 0.2 \\
\hline \multicolumn{8}{|c|}{ Premenopausal breast cancer } \\
\hline No of cases/non-cases & $264 / 57151$ & & $90 / 14263$ & $70 / 14284$ & $55 / 14299$ & 49/14305 & \\
\hline Model 1 & 1.09 (0.95 to 1.25$)$ & 0.2 & 1 & 0.91 (0.67 to 1.25$)$ & $0.92(0.65$ to 1.29$)$ & 1.30 (0.90 to 1.86$)$ & 0.3 \\
\hline Model 2 & $1.07(0.93$ to 1.23$)$ & 0.4 & 1 & $0.90(0.66$ to 1.24$)$ & $0.90(0.64$ to 1.27$)$ & $1.25(0.87$ to 1.80$)$ & 0.4 \\
\hline Model 3 & $1.09(0.95$ to 1.26$)$ & 0.2 & 1 & 0.91 (0.67 to 1.25$)$ & $0.92(0.66$ to 1.30$)$ & $1.30(0.91$ to 1.88$)$ & 0.3 \\
\hline Model 4 & $1.08(0.94$ to 1.24$)$ & 0.3 & 1 & 0.91 (0.66 to 1.24) & 0.91 (0.64 to 1.28$)$ & $1.27(0.88$ to 1.83$)$ & 0.4 \\
\hline \multicolumn{8}{|c|}{ Postmenopausal breast cancer } \\
\hline No of cases/non-cases & $475 / 29191$ & & $107 / 7309$ & $128 / 7289$ & $123 / 7294$ & $117 / 7299$ & \\
\hline Model 1 & $1.13(1.01$ to 1.27$)$ & 0.04 & 1 & $1.23(0.95$ to 1.60$)$ & $1.28(0.98$ to 1.66$)$ & 1.39 (1.07 to 1.82$)$ & 0.02 \\
\hline Model 2 & $1.13(1.00$ to 1.27$)$ & 0.05 & 1 & $1.23(0.95$ to 1.60$)$ & 1.27 (0.98 to 1.65$)$ & $1.39(1.05$ to 1.81$)$ & 0.02 \\
\hline Model 3 & $1.13(1.00$ to 1.27$)$ & 0.04 & 1 & 1.23 (0.95 to 1.59$)$ & 1.27 (0.98 to 1.65$)$ & $1.38(1.06$ to 1.81$)$ & 0.02 \\
\hline Model 4 & 1.13 (1.00 to 1.27$)$ & 0.05 & 1 & 1.23 (0.95 to 1.59$)$ & 1.27 (0.97 to 1.65$)$ & $1.38(1.05$ to 1.81$)$ & 0.02 \\
\hline
\end{tabular}

\section{HR=hazard ratio.}

*Model 1 =multivariable Cox proportional hazard model adjusted for age (timescale), sex, energy intake without alcohol, number of 24 hour dietary records, smoking status, educational level, physical activity, height, body mass index, alcohol intake, and family history of cancers; breast cancer models were additionally adjusted for menopausal status, hormonal treatment for menopause, oral contraception, and number of children. Model $2=$ model 1 plus intakes of lipids, sodium, and carbohydrates. Model $3=$ model 1 plus Western dietary pattern (derived by factor analysis). Model 4=model 1 plus intakes of lipids, sodium, and carbohydrates and Western dietary pattern (derived by factor analysis). Pearson correlation coefficients with Western dietary pattern were 0.5 for dietary lipids, 0.6 for sodium, and 0.40 for carbohydrates.

tHazard ratio for increase of $10 \%$ in proportion of ultra-processed food intake in diet.

¥Sex specific cut-offs for quarters of ultra-processed proportions were $11.8 \%, 16.8 \%$, and $23.3 \%$ in men and $11.8 \%, 16.8 \%$, and $23.4 \%$ in women. In premenopausal women, cut-offs were $12.8 \%, 18.1 \%$, and $25.0 \%$. In postmenopausal women, cut-offs were $10.1 \%, 14.3 \%$, and $19.5 \%$.

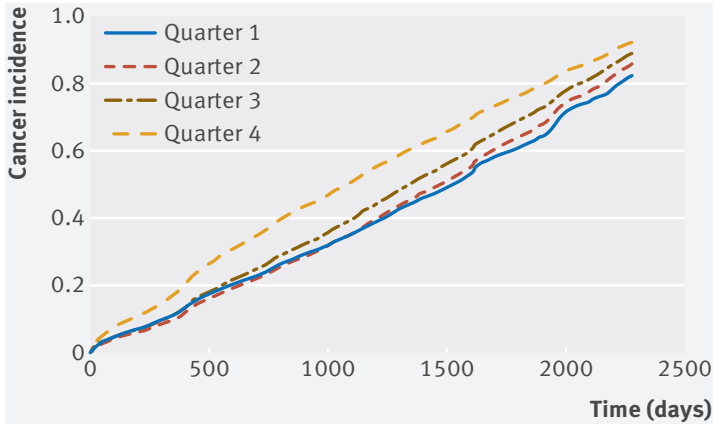

Fig 2 | Cumulative cancer incidence (overall cancer risk) according to quarters of proportion of ultra-processed food in diet
$(\mathrm{P}=0.005)$ were associated with an increased risk of overall cancer, and ultra-processed sugary products were associated with risk of breast cancer $(\mathrm{P}=0.006)$ (appendix 6).

Further adjustment for several indicators of the nutritional quality of the diet (lipid, sodium, and salt intakes-model 2; Western pattern-model 3; or both-model 4) did not modify these findings. The Pearson correlation coefficient between the proportion of ultra-processed food in the diet and the Western dietary pattern was low (0.06). Consistently, analyses performed according to the method proposed by Lange et al to assess a potential mediation of the relation between ultra-processed food and risk of cancer 
by these nutritional factors showed no statistically significant mediation effect of any of the factors tested. ${ }^{45}$ The mediated effects ranged between $0 \%$ and $2 \%$, with all $\mathrm{P}>0.05$ (appendix 4).

No association was statistically significant for prostate and colorectal cancers. However, we observed a borderline non-significant trend of increased risk of colorectal cancer associated with ultra-processed food intake (hazard ratio for quarter 4 versus quarter 1: 1.23 (1.08 to 1.40), P for trend=0.07) in model 4.

Sensitivity analyses (adjusted for model 1 covariates, data not tabulated) excluding cancer cases diagnosed during the first two years of follow-up provided similar results (hazard ratio for a 10 point increment in the proportion of ultra-processed foods in the diet 1.10 (1.03 to 1.17), $\mathrm{P}=0.005$ for overall cancer risk, 1367 cases and 102502 non-cases included; 1.15 (1.03 to 1.29), $\mathrm{P}=0.02$ for breast cancer risk, 441 cases and 80940 non-cases included). Similarly, results were unchanged when we excluded non-validated cancer cancers (hazard ratio for a 10 point increment in the proportion of ultra-processed foods in the diet 1.11 (1.05 to 1.17), $\mathrm{P}<0.001$ for overall cancer risk, 1967 cases and 102752 non-cases included; 1.12 (1.02 to 1.23), $\mathrm{P}=0.02$ for breast cancer risk, 677 cases and 81274 non-cases included).

We obtained similar results when we included only participants with at least six 24 hour records (overall cancer risk: hazard ratio for a 10 point increment in the proportion of ultra-processed foods in the diet 1.13 (1.06 to 1.21), $\mathrm{P}<0.001,1494$ cases and 47920 noncases included) and when we re-included participants with only one 24 hour record (overall cancer risk: 1.11 (1.06 to 1.16), $\mathrm{P}<0.001,2383$ cases and 122196 noncases included).

Findings were also similar when we coded the proportion of ultra-processed food in the diet as sex specific fifths instead of quarters (overall cancer risk: hazard ratio for highest versus lowest fifth 1.25 (1.08 to 1.47$), \mathrm{P}$ for trend $<0.001$; breast cancer risk: 1.25 (0.96 to 1.63), $P$ for trend $=0.03$ ).

Further adjustment for the following variables, in addition to model 1 covariates, did not modify the results: dietary supplement use at baseline (hazard ratio for a 10 point increment in the proportion of ultra-processed foods in the diet 1.12 (1.06 to 1.17), $\mathrm{P}<0.001$ for overall cancer; 1.11 (1.02 to 1.22 ), $\mathrm{P}=0.02$ for breast cancer), prevalent depression at baseline (1.11 (1.06 to 1.17), $\mathrm{P}<0.001$ for overall cancer; 1.11 (1.01 to 1.22), $\mathrm{P}=0.02$ for breast cancer), healthy dietary pattern (1.11 (1.05 to 1.17 ), $\mathrm{P}<0.001$ for overall cancer; 1.10 (1.00 to 1.21), $\mathrm{P}=0.04$ for breast cancer), overall fruit and vegetable consumption in $\mathrm{g} / \mathrm{d}$ (1.10 (1.04 to 1.16), $\mathrm{P}<0.001$ for overall cancer; 1.11 (1.01 to 1.22$), \mathrm{P}=0.03$ for breast cancer), number of smoked cigarettes in pack years (1.13 (1.07 to 1.19$), \mathrm{P}<0.001$ for overall cancer; 1.13 (1.03 to 1.24$), \mathrm{P}=0.009$ for breast cancer), and season of inclusion in the cohort (1.12 (1.06 to 1.18), $\mathrm{P}<0.001$ for overall cancer; 1.12 (1.02 to 1.22), $\mathrm{P}=0.02$ for breast cancer).
We also tested other methods for handling missing data, such as multiple imputation and complete case analysis (that is, exclusion of participants with missing data for at least one covariate). ${ }^{46}$ The results were very similar for the multiple imputation analysis (hazard ratio for a 10 point increment in the proportion of ultra-processed foods in the diet 1.11 (1.06 to 1.17), $\mathrm{P}<0.001,2228$ cases and 102752 non-cases for overall cancer; 1.11 (1.01 to 1.21 ), $\mathrm{P}=0.02,739$ cases and 81420 non-cases for breast cancer) and for the complete case analysis (1.11 (1.05 to 1.18), $\mathrm{P}<0.001$, 1813 cases and 82824 non-cases for overall cancer; 1.14 (1.03 to 1.26 ), $\mathrm{P}=0.01,579$ cases and 64642 non-cases for breast cancer).

As a secondary analysis, we also tested associations between the proportions of the three other NOVA degrees of food processing and risk of cancer. We found no significant associations between the proportions of "processed culinary ingredients" or "processed foods" with risk of cancer at any location (all P>0.05). However, and consistent with our findings, the consumption of "minimally/unprocessed foods" was associated with lower risks of overall and breast cancers (hazard ratio for a 10 point increment in the proportion of unprocessed foods in the diet 0.91 (0.87 to 0.95), $\mathrm{P}<0.001,2228$ cases and 102752 noncases for overall cancer; 0.42 ( 0.19 to 0.91$), \mathrm{P}=0.03$, 739 cases and 81420 non-cases for breast cancer), in multivariable analyses adjusted for model 1 covariates.

\section{Discussion}

In this large prospective cohort, a $10 \%$ increase in the proportion of ultra-processed foods in the diet was associated with significant increases of $12 \%$ in the risk of overall cancer and $11 \%$ in the risk of breast cancer. A few studies have previously suggested that ultra-processed foods contribute to increasing the risk of cardiometabolic disorders-such as obesity, ${ }^{29}$ hypertension, ${ }^{30}$ and dyslipidaemia ${ }^{28}$-but no previous prospective epidemiological study has evaluated the association between food processing and risk cancer.

\section{Interpretation and comparison with other studies}

No estimate is available of the proportion of ultraprocessed food in the diet at the national level in France. However, in the nationally representative INCA3 study conducted by the French Food safety Agency in 2016, "transformed" foods included sweet pastries, biscuits, dairy desserts, ice cream, fruit purée and fruit in syrup, fruit and vegetable juices, soups and broths, sandwiches, pizzas, and salted pastries, as well as mixed dishes composed of egg, meat, fish, vegetable, and/or starchy foods (cereals, legumes, or potatoes). More than half of the "transformed" foods consumed outside catering establishments by adults aged 18-79 were manufactured industrially, about a third were homemade, and the rest was handcrafted (for example, by caterers). These figures illustrate the important share of processed, and especially industrially processed, foods in the diet of French adults. 
Several hypotheses could be put forward to explain our findings. The first one relates to the generally poorer nutritional quality of diets rich in ultraprocessed foods. Diets that include a higher proportion of processed food products tended to be richer in energy, sodium, fat, and sugar and poorer in fibres and various micronutrients in several studies conducted in various countries. ${ }^{10-17} 19$ Ultra-processed foods have also been associated with a higher glycaemic response and a lower satiety effect. ${ }^{47}$ Although not the unique determinant, excessive energy, fat, and sugar intakes contribute to weight gain and risk of obesity, with obesity recognised as a major risk factor for post-menopausal breast, stomach, liver, colorectal, oesophagus, pancreas, kidney, gallbladder, endometrium, ovary, liver, and (advanced) prostate cancers and haematological malignancies. ${ }^{29}$ For instance, body fatness in post-menopausal women is estimated to contribute $17 \%$ of the breast cancer burden. $^{2}$ Furthermore, most ultra-processed foods, such as dehydrated soups, processed meats, biscuits, and sauces, have a high salt content. Foods preserved with salt are associated with an increased risk of gastric cancer. $^{29}$ Conversely, dietary fibre intake decreases the risk of colorectal cancer, with a convincing level of evidence, ${ }^{329}$ and may also reduce the risk of breast cancer. ${ }^{3}$ However, the associations between ultra-processed food intake and risk of cancer observed in this study were statistically significant despite adjustment for body mass index and remained significant after further adjustment for a Western-type dietary pattern and/or the energy, fat, sugar, and salt content of the diet. Mediation analyses did not support a strong effect of the "nutritional quality" component in this association, suggesting that other bioactive compounds contained in ultra-processed food may contribute to explain the observed associations.

A second hypothesis concerns the wide range of additives contained in ultra-processed foods. Although maximum authorised levels normally protect the consumers against adverse effects of each individual substance in a given food product, ${ }^{48}$ the effect on health of the cumulative intake across all ingested foods and potential cocktail/interaction effects remain largely unknown. More than 250 different additives are authorised for addition to food products in Europe and the US. ${ }^{22} 49$ For some of them, experimental studies in animal or cellular models have suggested carcinogenic properties that deserve further investigation in humans. ${ }^{23} 2450-53$ One example is titanium dioxide $\left(\mathrm{TiO}_{2}\right)$, a common food additive that contains nanoscale particles and that is used as a whitening agent or in packaging in contact with food or drinks to provide a better texture and antimicrobial properties. Experimental studies, mainly conducted in rodent models, suggest that this additive could initiate or promote the development of pre-neoplastic lesions in the colon, as well as chronic intestinal inflammation. The World Health Organization and the International Agency for Research on Cancer evaluated $\mathrm{TiO}_{2}$ as "possibly carcinogenic to humans" (group
2B). ${ }^{24}$ The effects of intense artificial sweeteners such as aspartame on human metabolism and on the composition and functioning of gut microbiota are also controversial. ${ }^{53}$ Although previous experimental studies in animals confirmed the safety of aspartame, their relevance to human health outcomes has been questioned, particularly regarding potential long term carcinogenicity. ${ }^{51}$ Another concern is the formation of carcinogenic nitrosamines in meats containing sodium nitrite when meat is charred or overcooked. These $\mathrm{N}$-nitroso compounds may be involved in causing colorectal cancer. ${ }^{23} 52$

Thirdly, food processing and particularly heat treatments produce neoformed contaminants (for example, acrylamide) in ultra-processed products such as fried potatoes, biscuits, bread, or coffee. A recent meta-analysis found a modest association between dietary acrylamide and risk of both kidney and endometrial cancer in non-smokers. ${ }^{54}$ In addition, the European Food Safety Agency judged that evidence from animal studies was sufficient to classify acrylamide as genotoxic. $^{20}$

Lastly, bisphenol A is another contaminant suspected of migrating from plastic packaging of ultraprocessed foods. Its endocrine disruptor properties led the European Chemicals Agency to judge it as "a substance of very high concern." ${ }^{55}$ Increasing evidence suggests involvement in the development of several non-communicable diseases, including cancer linked to endocrinal disruptors. ${ }^{21}$

\section{Strengths and limitations of study}

Strengths of this study pertain to its prospective design and large sample size, along with a detailed and up to date assessment of dietary intake. Repeated 24 hour dietary records (including 3300 different food items) are more accurate than either food frequency questionnaires with aggregated food groups or household purchasing data. However, some limitations should be acknowledged. Firstly, as is generally the case in volunteer based cohorts, participants in the NutriNet-Santé cohort were more often women, with health conscious behaviours and higher socio-professional and educational levels than the general French population. ${ }^{56}$ This might limit the generalisability of the findings and may have resulted in a lower incidence of cancer compared with national estimates (age and sex standardised incidence rate per 100000 people per year: 786 cases in our cohort versus 972 cases in France ${ }^{57}$ ) and an overall lower exposure to ultra-processed foods, with less contrast between extreme categories. These points would tend to lead to underestimation of the strength of the associations. However, the possibility that selection bias may have led to an overestimation of some associations cannot be totally excluded. Secondly, some misclassification in the NOVA "ultra-processed food" category cannot be ruled out. Thirdly, despite a multi-source strategy for case ascertainment (combining validation of health events declared by participants, medicoadministrative databases from the health insurance, 
and national death registry), exhaustive detection of cancer cases cannot be guaranteed. Furthermore, statistical power was limited for some cancer locations (such as colorectal cancer), which may have impaired our ability to detect hypothesised associations. Next, the length of follow-up was relatively limited, as the cohort was launched in 2009. It allowed us to study mostly mid-term associations between consumption of ultra-processed food and risk of cancer. As is usually the case in nutritional epidemiology, we made the assumption that the measured exposure at baseline (especially as we averaged a two year period of exposure) actually reflects more generally the usual eating habits of the individual during adulthood, including several years before his or her entry into the cohort. However, as some carcinogenic processes may take several decades, it will be important in the future to reassess the associations between ultraprocessed food and cancer risk in the cohort, to investigate longer term effects. This will be one of the perspectives of our work for the upcoming five to 10 years. Lastly, although we included a large range of confounding factors in the analyses, the hypothesis of residual confounding resulting from unmeasured behavioural factors and/or imprecision in the measure of included covariates cannot be entirely excluded owing to the observational design of this study. For instance, oral contraception was a binary variable in breast cancer models, as the precise doses, type, and duration of contraceptive use across reproductive life were not available. Randomised controlled trials have long been considered the only gold standard for elimination of confounding bias, but they do not capture consumption as it is in daily life. Moreover, a trial to investigate exposure for which a deleterious effect is suspected would not be ethically feasible. Our large observational cohort was therefore particularly adapted to provide insights in this field.

\section{Conclusions and policy implications}

To our knowledge, this study was the first to investigate and highlight an increase in the risk of overall-and more specifically breast-cancer associated with ultra-processed food intake. These results should be confirmed by other large scale, population based observational studies in different populations and settings. Further studies are also needed to better understand the relative effect of nutritional composition, food additives, contact materials, and neoformed contaminants in this relation. Rapidly increasing consumption of ultra-processed foods may drive an increasing burden of cancer and other non-communicable diseases. Thus, policy actions targeting product reformulation, taxation, and marketing restrictions on ultra-processed products and promotion of fresh or minimally processed foods may contribute to primary cancer prevention. ${ }^{6} 9$ Several countries have already introduced this aspect in their official nutritional recommendations in the name of the precautionary principle. ${ }^{5859}$
We sincerely thank all the volunteers of the NutriNet-Santé cohort. We also thank Véronique Gourlet, Lucien Martinez, Nathalie Arnault, Stephen Besseau, Laurent Bourhis, Yasmina Chelghoum, Than Duong Van, Younes Esseddik, Paul Flanzy, Julien Allègre, Mac Rakotondrazafy, Régis Gatibelza, Fabien Szabo, Roland Andrianasolo, Fatoumata Diallo, Ludivine Ursule, Cédric Agaesse, Claudia Chahine, Anne-Elise Dussoulier, and Marion Genest for their technical contribution to the NutriNet-Santé study.

Contributors: TF and BS contributed equally and are co-first authors. TF, BS, CJ, EKG, CAM, BA, and MT designed the research. SH, MT, $\mathrm{Cl}$, and EKG conducted the research. TF did the statistical analysis, supervised by MT and BS. TF and MT wrote the paper. BS did sensitivity analyses and was in charge of the revision of the paper. All authors contributed to the data interpretation, revised each draft for important intellectual content, and read and approved the final manuscript. MT is the guarantor.

Funding: The NutriNet-Santé study was supported by the following public institutions: Ministère de la Santé, Institut de Veille Sanitaire (InVS), Institut National de la Prévention et de l'Education pour la Santé (INPES), Région Ile-de-France (CORDDIM), Institut National de la Santé et de la Recherche Médicale (INSERM), Institut National de la Recherche Agronomique (INRA), Conservatoire National des Arts et Métiers (CNAM), and Université Paris 13. MD and PF were funded by a PhD grant from the Cancéropôle Ile de France/Région lle de France (public funding). BS was funded by the French National Cancer Institute (grant number INCa_8085). Researchers were independent from funders. Funders had no role in the study design; the collection, analysis, and interpretation of data; the writing of the report; or the decision to submit the article for publication.

Competing interests: All authors have completed the ICMJE uniform disclosure form at www.icmje.org/coi_disclosure.pdf (available on request from the corresponding author) and declare: no support from any organisation for the submitted work other than that described above; no financial relationships with any organisations that might have an interest in the submitted work in the previous three years; no other relationships or activities that could appear to have influenced the submitted work.

Ethical approval: The NutriNet-Santé study was approved by the Institutional Review Board of the French Institute for Health and Medical Research (IRB Inserm No 0000388FWA00005831) and the Commission Nationale de l'Informatique et des Libertés (CNIL No 908450/No 909216). Electronic informed consent was obtained from each participant.

Transparency statement: MT (the guarantor) affirms that the manuscript is an honest, accurate, and transparent account of the study being reported; that no important aspects of the study have been omitted; and that any discrepancies from the study as planned (and, if relevant, registered) have been explained.

Data sharing: No additional data available.

This is an Open Access article distributed in accordance with the Creative Commons Attribution Non Commercial (CC BY-NC 4.0) license, which permits others to distribute, remix, adapt, build upon this work non-commercially, and license their derivative works on different terms, provided the original work is properly cited and the use is non-commercial. See: http://creativecommons.org/licenses/ by-nc/4.0/.

1 Ferlay J, Soerjomataram I, Dikshit R, et al. Cancer incidence and mortality worldwide: sources, methods and major patterns in GLOBOCAN 2012. Int J Cancer 2015;136:E359-86. doi:10.1002/ ijc. 29210

2 World Cancer Research Fund International/American Institute for Cancer Research. Cancer preventability estimates for diet, nutrition, body fatness, and physical activity. 2017. wcrf.org/cancerpreventability-estimates.

3 Latino-Martel P, Cottet V, Druesne-Pecollo N, et al. Alcoholic beverages, obesity, physical activity and other nutritional factors, and cancer risk: A review of the evidence. Crit Rev Oncol Hematol 2016;99:308-23. doi:10.1016/j.critrevonc.2016.01.002

4 ANSES (French Agency for Food, Environmental and Occupational Health \& Safety). Étude individuelle nationale des consommations alimentaires 3 (INCA 3). 2017. https://www.anses.fr/fr/content/ inca-3-evolution-des-habitudes-et-modes-de-consommation-denouveaux-enjeux-en-mati\%C3\%A8re-de.

5 Monteiro CA, Moubarac JC, Cannon G, Ng SW, Popkin B. Ultraprocessed products are becoming dominant in the global food system. Obes Rev 2013;14(Suppl 2):21-8. doi:10.1111/ obr.12107 
6 Moodie R, Stuckler D, Monteiro C, et al, Lancet NCD Action Group. Profits and pandemics: prevention of harmful effects of tobacco, alcohol, and ultra-processed food and drink industries. Lancet 2013;381:670-9. doi:10.1016/S0140-6736(12)62089-3

7 Moubarac JC, Batal M, Martins AP, et al. Processed and ultraprocessed food products: consumption trends in Canada from 1938 to 2011. Can J Diet Pract Res 2014;75:15-21 doi:10.3148/75.1.2014.15

8 Venn D, Banwell C, Dixon I. Australia’s evolving food practices: a risky mix of continuity and change. Public Health Nutr 2017;20:2549-58. doi:10.1017/S136898001600255X

9 Monteiro CA, Cannon G, Moubarac JC, Levy RB, Louzada MLC, Jaime PC. The UN Decade of Nutrition, the NOVA food classification and the trouble with ultra-processing. Public Health Nutr 2018;21:5 17. doi:10.1017/S1368980017000234

10 Luiten CM, Steenhuis IH, Eyles H, Ni Mhurchu C, Waterlander WE. Ultra-processed foods have the worst nutrient profile, yet they are the most available packaged products in a sample of New Zealand supermarkets--CORRIGENDUM. Public Health Nutr 2016;19:539. doi:10.1017/S1368980015002840

11 Adams J, White M. Characterisation of UK diets according to degree of food processing and associations with socio-demographics and obesity: cross-sectional analysis of UK National Diet and Nutrition Survey (2008-12). Int J Behav Nutr Phys Act 2015;12:160. doi:10.1186/s12966-015-0317-y

12 Cediel G, Reyes M, da Costa Louzada ML, et al. Ultra-processed foods and added sugars in the Chilean diet (2010). Public Health Nutr 2018;21:125-33. doi:10.1017/S1368980017001161

13 Costa Louzada ML, Martins AP, Canella DS, et al. Ultra-processed foods and the nutritional dietary profile in Brazil. Rev Saude Publica 2015;49:38

14 Martínez Steele E, Baraldi LG, Louzada ML, Moubarac JC, Mozaffarian D, Monteiro CA. Ultra-processed foods and added sugars in the US diet: evidence from a nationally representative cross-sectional study. BMJ Open 2016;6:e009892. doi:10.1136/ bmjopen-2015-009892

15 Moubarac JC, Martins AP, Claro RM, Levy RB, Cannon G, Monteiro CA Evidence from Canada. Consumption of ultra-processed foods and likely impact on human health. Public Health Nutr 2013;16:2240-8. doi:10.1017/S1368980012005009

16 Moubarac JC, Batal M, Louzada ML, Martinez Steele E, Monteiro CA. Consumption of ultra-processed foods predicts diet quality in Canada. Appetite 2017;108:512-20. doi:10.1016/j. appet.2016.11.006

17 Poti JM, Mendez MA, Ng SW, Popkin BM. Is the degree of food processing and convenience linked with the nutritional quality of foods purchased by US households? Am J Clin Nutr 2015;101:1251 62. doi:10.3945/ajcn.114.100925

18 Slimani N, Deharveng G, Southgate DA, et al. Contribution of highly industrially processed foods to the nutrient intakes and patterns of middle-aged populations in the European Prospective Investigation into Cancer and Nutrition study. Eur J Clin Nutr 2009;63(Suppl 4):S206-25. doi:10.1038/ejcn.2009.82

19 Louzada ML, Martins AP, Canella DS, et al. Impact of ultra-processed foods on micronutrient content in the Brazilian diet. Rev Saude Publica 2015;49:45.

20 Panel on Contaminants in the Food Chain. Acrylamide in food. EFSA Journal 2015;13:4104doi:10.2903/j.efsa.2015.4104.

21 MunckeJ. Endocrine disrupting chemicals and other substances of concern in food contact materials: an updated review of exposure, effect and risk assessment. / Steroid Biochem Mol Biol 2011:127:118-27. doi:10.1016/j.jsbmb.2010.10.004

22 European Union. Database of authorized food additives. 2008. https://ec.europa.eu/food/safety/food_improvement_agents/ additives/database_en.

23 Bouvard V, Loomis D, Guyton KZ, et al, International Agency for Research on Cancer Monograph Working Group. Carcinogenicity of consumption of red and processed meat. Lancet Oncol 2015:16:1599-600 doi:10.1016/S1470-2045(15)00444-1

24 IARC Working Group on the Evaluation of Carcinogenic Risks to Humans. Carbon black, titanium dioxide, and talc. IARC Monogr Eval Carcinog Risks Hum 2010;93:1-413.

25 Canella DS, Levy RB, Martins AP, et al. Ultra-processed food products and obesity in Brazilian households (2008-2009). PLoS One 2014;9:e92752. doi:10.1371/journal.pone.009275

26 Juul F, Hemmingsson E. Trends in consumption of ultra-processed foods and obesity in Sweden between 1960 and 2010. Public Health Nutr 2015;18:3096-107. doi:10.1017/S1368980015000506

27 Louzada ML, Baraldi LG, Steele EM, et al. Consumption of ultraprocessed foods and obesity in Brazilian adolescents and adults. Prev Med 2015;81:9-15. doi:10.1016/j.ypmed.2015.07.018

28 Rauber F, Campagnolo PD, Hoffman DJ, Vitolo MR. Consumption of ultra-processed food products and its effects on children's lipid profiles: a longitudinal study. Nutr Metab Cardiovasc Dis 2015;25:116-22. doi:10.1016/j.numecd.2014.08.001
29 Mendonça RD, Pimenta AM, Gea A, et al. Ultraprocessed food consumption and risk of overweight and obesity: the University of Navarra Follow-Up (SUN) cohort study. Am J Clin Nutr 2016;104:1433-40. doi:10.3945/ajcn.116.135004

30 Mendonça RD, Lopes AC, Pimenta AM, Gea A, MartinezGonzalez MA, Bes-Rastrollo M. Ultra-Processed Food Consumption and the Incidence of Hypertension in a Mediterranean Cohort: The Seguimiento Universidad de Navarra Project. Am J Hypertens 2017:30:358-66.

31 Hercberg S, Castetbon K, Czernichow S, et al. The Nutrinet-Santé Study: a web-based prospective study on the relationship between nutrition and health and determinants of dietary patterns and nutritional status. BMC Public Health 2010;10:242. doi:10.1186/1471-2458-10-242

32 Vergnaud AC, Touvier M, Méjean C, et al. Agreement between webbased and paper versions of a socio-demographic questionnaire in the NutriNet-Santé study. Int J Public Health 2011;56:407-17. doi:10.1007/s00038-011-0257-5

33 Lassale C, Péneau S, Touvier M, et al. Validity of web-based selfreported weight and height: results of the Nutrinet-Santé study. J Med Internet Res 2013;15:e152. doi:10.2196/jmir.2575

34 Touvier M, Méjean C, Kesse-Guyot E, et al. Comparison between web-based and paper versions of a self-administered anthropometric questionnaire. Eur J Epidemiol 2010;25:287-96. doi:10.1007/ s10654-010-9433-9

35 Craig CL, Marshall AL, Sjöström M, et al. International physical activity questionnaire: 12 -country reliability and validity. Med Sci Sports Exerc 2003;35:1381-95. doi:10.1249/01. MSS.0000078924.61453.FB

36 Touvier M, Kesse-Guyot E, Méjean C, et al. Comparison between an interactive web-based self-administered $24 \mathrm{~h}$ dietary record and an interview by a dietitian for large-scale epidemiological studies. Br J Nutr 2011;105:1055-64. doi:10.1017/ S0007114510004617

37 Lassale C, Castetbon K, Laporte F, et al. Validation of a Web-based, self-administered, non-consecutive-day dietary record tool against urinary biomarkers. Br J Nutr 2015;113:953-62. doi:10.1017/ S0007114515000057

38 Lassale C, Castetbon K Laporte F et al. Correlations between Fruit, Vegetables, Fish, Vitamins, and Fatty Acids Estimated by Web Based Nonconsecutive Dietary Records and Respective Biomarkers of Nutritional Status. J Acad Nutr Diet 2016;116:427-438.e5. doi:10.1016/j.jand.2015.09.017

39 Le Moulenc N, Deheeger M, Preziozi P, et al. Validation du manuel photo utilisé pour l'enquête alimentaire de l'étude SU.VI. MAX[Validation of the food portion size booklet used in the SU.VI. MAX study]. Cahiers de Nutrition et de Diététique 1996;31:158-64.

40 Black AE. Critical evaluation of energy intake using the Goldberg cut-off for energy intake:basal metabolic rate. A practical guide to its calculation, use and limitations. Int J Obes Relat Metab Disord 2000;24:1119-30. doi:10.1038/sj.ijo.0801376

41 Etude Nutrinet Santé. Table de composition des aliments, étude NutriNet-Santé [Food composition table, NutriNet-Santé study] . Les éditions INSERM/Economica, 2013.

42 Monteiro CA, Cannon G, Levy RB, et al. NOVA. The star shines bright. World Nutrition 2016;7:28-38.

43 Moubarac JC, Parra DC, Cannon G, Monteiro CA. Food Classification Systems Based on Food Processing: Significance and Implications for Policies and Actions: A Systematic Literature Review and Assessment. Curr Obes Rep 2014;3:256-72. doi:10.1007/s13679014-0092-0

44 Julia C, Martinez L, Allès B, et al. Contribution of ultra-processed foods in the diet of adults from the French NutriNet-Santé study. Public Health Nutr 2018;21:27-37. doi:10.1017/S1368980017001367

45 Lange T, Vansteelandt S, Bekaert M. A simple unified approach for estimating natural direct and indirect effects. Am Epidemiol 2012;176:190-5. doi:10.1093/aje/kwr525

46 Sterne JA, White IR, Carlin JB, et al. Multiple imputation for missing data in epidemiological and clinical research: potential and pitfalls. BMJ 2009;338:b2393. doi:10.1136/bmj.b2393

47 Fardet A. Minimally processed foods are more satiating and less hyperglycemic than ultra-processed foods: a preliminary study with 98 ready-to-eat foods. Food Funct 2016;7:2338-46. doi:10.1039/ C6F000107F

48 World Health Organization. Food additives. 2017. http://www.who. int/mediacentre/factsheets/food-additives/en/.

49 US Food and Drug Administration. Food additive status list. 2017. https://www.fda.gov/food/ingredientspackaginglabeling/ foodadditivesingredients/ucm091048.htm

50 Chang X, Zhang Y, Tang M, Wang B. Health effects of exposure to nano-TiO2: a meta-analysis of experimental studies. Nanoscale Res Lett 2013;8:51. doi:10.1186/1556-276X-8-51

51 Panel on Food Additives and Nutrient Sources Added to Food. Opinion on the re-evaluation of aspartame (E 951) as a food additive EFSA lournal 2013:11:3496. 
52 Santarelli RL, Vendeuvre JL, Naud N, et al. Meat processing and colon carcinogenesis: cooked, nitrite-treated, and oxidized high-heme cured meat promotes mucin-depleted foci in rats. Cancer Prev Res (Phila) 2010;3:852-64. doi:10.1158/1940-6207.CAPR-09-0160

53 Suez J, Korem T, Zeevi D, et al. Artificial sweeteners induce glucose intolerance by altering the gut microbiota. Nature 2014;514:181-6. doi:10.1038/nature13793

54 Virk-Baker MK, Nagy TR, Barnes S, Groopman J. Dietary acrylamide and human cancer: a systematic review of literature. Nutr Cancer 2014;66:774-90. doi:10.1080/01635581.2014. 916323

55 European Chemical Agency (ECHA). Member State Committee support document for identification of 4,4'-isopropylidenediphenol (bisphenol a) as a substance of very high concern because of its toxic for reproduction (Article $57 \mathrm{c}$ ) properties. Adopted on 2 December 2016. https://echa.europa.eu/documents/10162/b10d6a00-8e479b14-4f61-c779a8dc8450.
56 Andreeva VA, Salanave B, Castetbon K, et al. Comparison of the sociodemographic characteristics of the large NutriNetSante e-cohort with French Census data: the issue of volunteer bias revisited. J Epidemiol Community Health 2015;69:893-8. doi:10.1136/jech-2014-205263

57 Institut National du Cancer. Les Cancer en France. 2014. http:// www.unicancer.fr/sites/default/files/Les $\% 20$ cancers $\% 20$ en $\% 20$ France\%20-\%20Edition\%202014\%20-\%20V5.pdf.

58 Ministry of Health of Brazil. Dietary guidelines for the Brazilian population. Ministry of Health of Brazil, 2014.

59 Haut Conseil de la Santé Publique. Avis relatif à la révision des repères alimentaires pour les adultes du futur Programme National Nutrition Santé 2017-2021, 2017. https://www.hcsp.fr/Explore.cgi/ avisrapportsdomaine?clefr $=600$.

\section{Appendix 1-6}

